Modern Physics Letters A

(C) World Scientific Publishing Company

\title{
HIGH ENERGY NEUTRINOS FROM A SLOW JET MODEL OF CORE COLLAPSE SUPERNOVAE
}

\author{
SOEBUR RAZZAQUE \\ Department of Astronomy and Astrophysics, Pennsylvania State University \\ 525 Davey Laboratory, University Park, Pennsylvania 16802, USA \\ soeb@astro.psu.edu \\ PETER MÉSZÁROS \\ Department of Astronomy and Astrophysics, Department of Physics \\ Pennsylvania State University \\ 525 Davey Laboratory, University Park, Pennsylvania 16802, USA \\ ELI WAXMAN \\ Physics Faculty, Weizman Institute of Science \\ Rehovot 76100, Israel
}

\begin{abstract}
It has been hypothesized recently that core collapse supernovae are triggered by mildly relativistic jets following observations of radio properties of these explosions. Association of a jet, similar to a gamma-ray burst jet but only slower, allows shock acceleration of particles to high energy and non-thermal neutrino emission from a supernova. Detection of these high energy neutrinos in upcoming kilometer scale Cherenkov detectors may be the only direct way to probe inside these astrophysical phenomena as electromagnetic radiation is thermal and contains little information. Calculation of high energy neutrino signal from a simple and slow jet model buried inside the pre-supernova star is reviewed here. The detection prospect of these neutrinos in water or ice detector is also discussed in this brief review. Jetted core collapse supernovae in nearby galaxies may provide the strongest high energy neutrino signal from point sources.
\end{abstract}

Keywords: supernovae; neutrinos; jet; core-collapse, gamma-rays: burst

\section{Introduction}

Core collapse of massive stars which lead to supernovae (SNe) of type Ib,c and II are in some cases associated with long duration ( $\gtrsim 2-10^{3} \mathrm{~s}$ ) gamma-ray bursts (GRBs), as evidenced by observed correlations of GRB 980425/SN 1998bw, GRB 021211/SN 2002lt, GRB 030329/SN 2003dh and GRB 0131203/SN 20031w! 1 A relativistic jet with bulk Lorentz factor $\Gamma_{b} \gtrsim 100$, powered by a black hole and an accretion disc which form after the core collapse in the most likely scenario, is believed to lead to the GRB event ${ }^{2}$ Observational evidence of only a small fraction of detected SNe associated with GRBs hints that the frequency of highly relativistic jets in core collapse $\mathrm{SNe}$ is at best 1 in 1000 , roughly the ratio of GRB to SN rates 
However, a significantly larger fraction $\left(\lesssim 10 \%\right.$ of type $\mathrm{Ib} / \mathrm{c}$ rate ${ }^{45}$ ) of $\mathrm{SNe}$ (also called hypernovae) may have mildly relativistic jets associated with them. 6789 One or more of the following observations support the jetted SN hypothesis:

- High expansion velocity $(30-40 \times 1000 \mathrm{~km} / \mathrm{s})$ first observed in SN 1998bw 10

- Radio afterglow not associated with $\gamma$-ray emission.11

- Asymmetric explosion supported by polarimetry observations of SN type $\mathrm{Ib} / \mathrm{c} 1213$

Numerical simulations of core collapse SNe, carried out over the last three decades have failed to produce a successful explosion by a prompt shock wave created due to the collapse of its iron core 14 The deposition of bulk kinetic energy in a jet form into the stellar envelope may help disrupt and blow it up making the SN possible 156 The presence of a jet is also conducive to shock acceleration of particles. In case of a GRB, internal shocks of plasma material along the jet accelerate protons and electrons which radiate observed $\gamma$-rays 16 High energy protons may escape as cosmic rays and/or produce $100 \mathrm{TeV}$ neutrinos by interacting with $\gamma$-rays in situ ${ }^{17}$ While the GRB jet is making its way out of the collapsing stellar progenitor it is expected to produce $10 \mathrm{TeV}$ neutrio precursor burst 1819 These neutrinos are emitted even in the cases when the jets do not manage to burrow through the stellar envelope and choke inside without producing observable $\gamma$-rays. The jets in core collapse $\mathrm{SNe}$ or hypernovae which is the topic of this review are slow with $\Gamma_{b} \sim$ few and choke inside the stellar envelope. ${ }^{20}$ Neutrinos produce from such jets are typically of a hundred $\mathrm{GeV}$ to $\mathrm{TeV}$ energy 2021

As opposed to $10 \mathrm{MeV}$ thermal neutrinos produced by the core collapse SN shocks which have been detected from SN $1987 \mathrm{~A}$ in our own galaxy, ${ }^{22}$ a high energy neutrinos from the jets may be detected from a longer distance because of an increasing detection prospect with neutrino energy. Kilometer scale ice and water Cherenkov detectors such as IceCube 24 and ANTARES 25 which are currently being built in Antarctica and in the Mediterranean will have an excellent chance to detect these neutrinos from SNe within the nearest $20 \mathrm{Mpc}$.

The organization of this brief review is as follows: In Sec. 2] a basic core collapse SN picture is outlined and a particular slow jet model in Sec. 3 Shock acceleration and the maximum energy reachable by protons are discussed in Sec. 4 Neutrino flux on Earth from a point source and diffuse sources is calculated in Sec. $\mathbf{5}$ and their detection prospects in Sec. 6] Conclusions are given in Sec. [7]

\section{Core Collapse Scenario}

Nuclear fusion reactions, similar to the ones which take place in our sun, constantly enrich the interior of a star forming an iron core as the end product. Burning up all fusion materials causes hydrodynamic instability due to lack of radiation pressure

${ }^{a}$ see Ref. 23 for a review of current experimental status to detect these low energy neutrinos. 
from inside the star. The immense gravitational pressure of the stellar envelope and/or overlying material causes the core of stars with mass $\gtrsim 8 M_{\odot}$ to collapse at this point. The density of the compressed core material reaches a few times the nuclear density and a rising temperature helps iron dissociate into nucleons and alpha particles.

Infall of stellar material onto the core produces $\sim 10 \mathrm{MeV}$ neutrinos by the process of electron capture on protons $\left(e^{-} p \rightarrow \nu_{e} n\right)$. The density of neutrons in the core exceeds that of protons in this process, called neutronization. Initially the neutrinos are trapped within a radius called neutrinosphere because of a density $\gtrsim 10^{12} \mathrm{~g}-\mathrm{cm}^{-3}$. For progenitors of mass $\lesssim 28 M_{\odot}$, the increasing degeneracy pressure of the neutrons leads to a rebound, which sends a shockwave through the core. While traversing through the core, the shockwave heats up material, dissociates more iron atoms and releases trapped $\nu_{e}$ from the neutrinosphere. Neutrinos carry away $\lesssim 10^{52}$ erg of energy or roughly $1 \%$ of the total gravitational binding energy in this bursting phase which lasts for a few milliseconds. The shockwave, however, does not reach the envelope to drive it away because of heavy energy loss and the star fails to explode into a supernova.

The mechanisms envisaged to produce a successful supernova explosion, such as observed in nature, may be divided into two main categories despite many uncertainties such as the mass loss rate of the pre-supernova star and neutrino transport in the core, to name a few. The first is a revived shock model, for stars initially less massive than $\sim 28 M_{\odot}$, where the core collapses to make a neutron star. In this case, the above-mentioned stalled supernova shock is re-energized by neutrino absorption on nucleons outside the stellar core $\left(\bar{\nu}_{e} p \rightarrow e^{+} n ; \nu_{e} n \rightarrow e^{-} p\right)$, re-energizing them. The shock wave then reaches the envelope and expels it away. After the supernova explosion, the stellar core cools down in next 10's of seconds by emitting $\sim 3 \times 10^{53}$ erg of energy in neutrinos of all flavors created by lepton pair annihilation $\left(e^{+} e^{-} \rightarrow \nu_{\mu, \tau} \bar{\nu}_{\mu, \tau}\right)$, neutrino pair annihilation $\left(\nu_{e} \bar{\nu}_{e} \rightarrow \nu_{\mu, \tau} \bar{\nu}_{\mu, \tau}\right)$ and nucleon bremsstrahlung $\left(N N \rightarrow N N \nu_{\mu, \tau} \bar{\nu}_{\mu, \tau}\right)$. These neutrinos are thermal with a typical energy $\sim 10 \mathrm{MeV}$. A neutron star is left over after the stellar core cools down, following ejection of the envelope and outer core.

In the second scenario, which is the relevant case for the current review, a star initially more massive than $\sim 28 M_{\odot}$ undergoes core collapse. (a) If the initial mass is $28 M_{\odot} \lesssim M_{*} \lesssim 40 M_{\odot}$, the core collapses initially to a neutron star, but after fallback of additional core gas which did not reach ejection velocity, it collapses further to a black hole $(\mathrm{BH})$ of mass in excess of the Chandrasekhar mass (the neutron degeneracy pressure not being sufficient to counteract gravity for this mass). (b) If the initial mass $M_{*} \gtrsim 40 M_{\odot}$, the core collapses directly to a black hole of mass $\gtrsim 3-5 M_{\odot}$ (see Ref. 26 e.g.). Thermal neutrinos of $\sim 10-30 \mathrm{MeV}$ are also produced in the neutronized core, before it falls into the black hole. This neutrino luminosity, of order several solar luminosities, may or may not be able to eject (via absorption in re-energization) the outer envelope, which is needed in order for it to appear as supernova detectable by its photon emission. As in the case of stars of mass 
$\lesssim 28 M_{\odot}$, numerical simulations have not yet been able to prove whether stars in this mass range eject their envelope; and, observationally, it is unclear whether any observed supernova can be ascribed to progenitors in this mass range. However, stars more massive than $\sim 28 M_{\odot}$ are certainly observed, and from well understood physics, they must core-collapse to a black hole. This is the gist of what happens to stars of mass $\gtrsim 28 M_{\odot}$ if the stellar core is rotating slowly. A black hole, and possibly a temporary small accretion disk is formed after core collapse, which may not greatly affect the symmetry of the collapse and/or envelope ejection.

The situation is thought to be drastically different for stars in the range $M_{*} \gtrsim$ $28 M_{\odot}$ whose core is fast-rotating ${ }^{27]}$ In particular, for a core angular momentum in the range $(3-20) \times 10^{16} \mathrm{~cm}^{2} \mathrm{~s}^{-1}$ for this model, the black hole and disk accretion can serve as an energy source for powering a long GRB, and may be able to eject a stellar envelope with kinetic energies possibly 10-100 times higher (at least in an isotropic-equivalent sense) than the energy of typical type Ib/c or type II SNe. Fast core rotation also helps in forming low density channels along the rotation axis, by centrifugal evacuation. Part of the material accreting from the disc onto the black hole can then be ejected as narrow jets along the axes, collimated by the gas pressure of the envelope. The jets are powered by neutrino annihilation or magnetohydrodynamic stresses. The pressure and energy deposition from the jet helps eject the star's envelope and thus the SN explosion happens. In the case of a highly relativistic jet which manages to break through the stellar envelope, the GRB event takes place outside the star in an optically thin environment. A slow jet, which is modelled in the next section, may never break through, but the ejected material can give rise to an "orphan" radio afterglow which is not associated with $\gamma$-ray emission. However, non-thermal high energy $(\sim \mathrm{TeV})$ neutrinos are produced ubiquitously in both cases.

\section{A Slow Jet Model}

The pre-supernova star, after losing its outermost envelope, is typically a WolfRayet star of radius $\sim 10^{11} \mathrm{~cm}$ in the case of a type $\mathrm{Ib}$ or larger in the case of a type II supernova. The mildly relativistic SN jet may be modelled inside the pre-supernova star (see Fig. (1) with a bulk Lorentz factor $\Gamma_{b} \sim 10^{0.5} \Gamma_{b, 0.5}$ and a total jet kinetic energy $E_{j} \sim 10^{51.5} E_{51.5}$ ergs which is $\sim 1 \%$ of the total energy released in the SN explosion. Because of its relativistic motion, the jet is most likely beamed with an opening half angle $\theta_{j} \sim 1 / \Gamma_{b}$ which is much wider compared to a GRB jet of $\Gamma_{b} \gtrsim 100$. Assuming a duration $t_{j} \sim 10 t_{j, 1} \mathrm{~s}$, the isotropic equivalent kinetic luminosity of the jet is $L_{\text {kin }} \simeq 2 \Gamma_{b}^{2} E_{j} / t_{j}$. With a jet variability time scale $t_{v} \sim 0.1 t_{v,-1} \mathrm{~s}$ at the base, internal shocks between plasma materials moving along the jet occur at a radius $r_{j} \simeq 2 \Gamma_{b}^{2} c t_{v} \approx 10^{10.8} \Gamma_{b, 0.5}^{2} t_{v,-1} \mathrm{~cm}$, which is below the stellar surface.

Internal shocks convert a fraction $\varepsilon_{e} \sim 0.1 \varepsilon_{e,-1}$ of the bulk kinetic energy $\left(E_{j}\right)$ into random electron motion analogous to the GRB fireball models. In the case of 


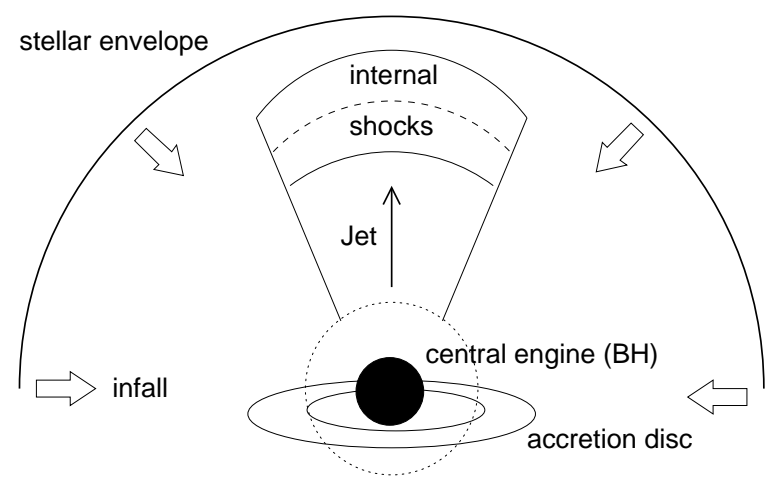

Fig. 1. Cartoon of a mildly relativistic jet buried inside the envelope of a collapsing star. The jet may deposit its kinetic energy and help blow up the envelope but may not get out itself.

GRBs, these relativistic electrons would emit synchrotron photons in an optically thin environment, which are observed as $\gamma$-rays on Earth. The density of these electrons and baryons (since they are coupled to the baryons) in the jet may be estimated as

$$
n_{e}^{\prime} \simeq n_{p}^{\prime} \simeq \frac{L_{\mathrm{kin}}}{4 \pi r_{j}^{2} \Gamma_{b}^{2} m_{p} c^{3}} \simeq \frac{E_{j}}{2 \pi r_{j}^{2} m_{p} c^{3} t_{j}} \approx 10^{20.5} \frac{E_{51.5}}{\Gamma_{b, 0.5}^{4} t_{j, 1} t_{v,-1}^{2}} \mathrm{~cm}^{-3},
$$

in the comoving jet frame. ${ }^{\mathrm{b}}$ The opacity to Thomson scattering by photons

$$
\tau_{\mathrm{Th}}^{\prime} \simeq \frac{\sigma_{\mathrm{Th}} n_{e, p}^{\prime} r_{j}}{\Gamma_{b}} \approx 10^{6.6} \frac{E_{51.5}}{\Gamma_{b, 0.5}^{3} t_{j, 1} t_{v,-1}}
$$

in the comoving frame is then very high.

The magnetic fields in the jet, built up by turbulent motions in the shock region, have an energy characterized as a fraction $\varepsilon_{B} \sim 0.1 \varepsilon_{B,-1}$ of the total jet energy, $B^{\prime 2} / 8 \pi \simeq \varepsilon_{B} L_{\mathrm{kin}} / 4 \pi r_{j}^{2} \Gamma_{b}^{2} c$. The corresponding magnetic field strength in the jet is given by

$$
B^{\prime} \simeq\left(\frac{4 \varepsilon_{B} E_{j}}{r_{j}^{2} c t_{j}}\right)^{1 / 2} \approx 10^{9}\left(\frac{\varepsilon_{B,-1} E_{51.5}}{\Gamma_{b, 0.5}^{2} t_{j, 1} t_{v,-1}}\right)^{1 / 2} \mathrm{G} .
$$

Electrons and protons are expected to be accelerated to high energies in the internal shocks, via the Fermi mechanism. The electrons cool down rapidly by synchrotron radiation in the presence of the magnetic field in Eq. (3). However, due to the large Thomson optical depth in Eq. (2), these photons thermalize and the corresponding black-body temperature is

$$
E_{\gamma}^{\prime} \simeq\left(\frac{15(\hbar c)^{3} \varepsilon_{e} E_{j}}{2 \pi^{4} r_{j}^{2} c t_{j}}\right)^{1 / 4} \approx 4.3\left(\frac{\varepsilon_{e,-1} E_{51.5}}{\Gamma_{b, 0.5}^{4} t_{j, 1} t_{v,-1}^{2}}\right)^{1 / 4} \mathrm{keV}
$$

${ }^{\mathrm{b}}$ Hereafter the variables in the comoving and in the laboratory frame or local rest frame are denoted with and without a prime respectively. 
The volume number density of these thermal photons may be roughly calculated as

$$
n_{\gamma}^{\prime} \simeq \frac{\varepsilon_{e} E_{j}}{2 \pi r_{j}^{2} c E_{\gamma}^{\prime} t_{j}} \approx 10^{24.8}\left(\frac{\varepsilon_{e,-1}^{3} E_{51.5}^{3}}{\Gamma_{b, 0.5}^{12} t_{j, 1}^{3} t_{v,-1}^{6}}\right)^{1 / 4} \mathrm{~cm}^{-3} .
$$

It may be noted that photons from the shocked stellar plasma do not diffuse into the jet due to the high optical depth $\tau_{\text {Th }}^{\prime}$ in Eq. (2), and the number density in Eq. (5) is roughly constant.

\section{Proton Acceleration and Cooling Processes}

The shock acceleration time for a proton of energy $E_{p}^{\prime}$ is proportional to its Larmor's radius and may be estimated as

$$
t_{\mathrm{acc}}^{\prime} \simeq \frac{A E_{p}^{\prime}}{q c B^{\prime}} \approx 10^{-12}\left(\frac{E_{p}^{\prime}}{\mathrm{GeV}}\right)\left(\frac{\kappa_{1}^{2} \Gamma_{b, 0.5}^{4} t_{j, 1} t_{v,-1}^{2}}{\varepsilon_{B,-1} E_{51.5}}\right)^{1 / 2} \mathrm{~s},
$$

where $\kappa \sim 10 \kappa_{1}$ and the magnetic field in Eq. (3) was used. The maximum proton energy is limited by requiring this time not to exceed the dynamic time scale for the shock to cross plasma material: $t_{\mathrm{dyn}}^{\prime} \simeq t_{v} \Gamma_{b}$, or any other possible proton cooling process time scale which we discuss next.

\subsection{Electromagnetic cooling channels}

\subsubsection{Synchrotron and inverse Compton}

The cooling time scale for protons by synchrotron radiation in the same magnetic field which is responsible for its acceleration is given by

$$
t_{\mathrm{syn}}^{\prime} \simeq \frac{6 \pi m_{p}^{4} c^{3}}{\sigma_{\mathrm{Th}} \beta^{2} m_{e}^{2} E_{p}^{\prime} B^{\prime 2}} \approx 3.8\left(\frac{E_{p}^{\prime}}{\mathrm{GeV}}\right)^{-1}\left(\frac{\Gamma_{b, 0.5}^{4} t_{j, 1} t_{v,-1}^{2}}{\varepsilon_{B,-1} E_{51.5}}\right) \mathrm{s},
$$

with $\beta=v / c \sim 1$. Inverse Compton (IC) scattering of thermal electron synchrotron photons is another cooling channel for high energy protons. The IC cooling time scale in the Thomson and Klein-Nishina $(\mathrm{KN})$ regimes, valid for $E_{p}^{\prime}$ much less or greater than $m_{p}^{2} c^{4} / E_{\gamma}^{\prime} \approx 10^{5.3}\left(\Gamma_{b, 0.5}^{4} t_{j, 1} t_{v,-1}^{2} / E_{51.5} \varepsilon_{e,-1}\right)^{1 / 4} \mathrm{GeV}$ respectively, as

$$
\begin{gathered}
t_{\mathrm{IC}, \mathrm{Th}}^{\prime}=\frac{3 m_{p}^{4} c^{3}}{4 \sigma_{\mathrm{Th}} m_{e}^{2} E_{p}^{\prime} E_{\gamma}^{\prime} n_{\gamma}^{\prime}} \approx 3.8\left(\frac{E_{p}^{\prime}}{\mathrm{GeV}}\right)^{-1}\left(\frac{\Gamma_{b, 0.5}^{4} t_{j, 1} t_{v,-1}^{2}}{\varepsilon_{e,-1} E_{51.5}}\right) \mathrm{s} \\
t_{\mathrm{IC}, \mathrm{KN}}^{\prime}=\frac{3 E_{p}^{\prime} E_{\gamma}^{\prime}}{4 \sigma_{\mathrm{Th}} m_{e}^{2} c^{5} n_{\gamma}^{\prime}} \approx 10^{-10.5}\left(\frac{E_{p}^{\prime}}{\mathrm{GeV}}\right)\left(\frac{\Gamma_{b, 0.5}^{4} t_{j, 1} t_{v,-1}^{2}}{\varepsilon_{e,-1} E_{51.5}}\right)^{1 / 2} \mathrm{~s} .
\end{gathered}
$$

Here we used the thermal photons with peak energy and density given in Eqs. (4) and (5) respectively as targets. 


\subsubsection{Bethe-Heitler}

Because of a high density of thermal photons in the SN jet in Eq. (5), protons may produce $e^{+} e^{-}$pairs by interacting with them, a process known as BetheHeitler (BH). The cross-section for $\mathrm{BH}$ interaction: $p \gamma \rightarrow p e^{+} e^{-}$is given by $\sigma_{\mathrm{BH}}=$ $\alpha r_{e}^{2}\left\{(28 / 9) \ln \left[2 E_{p}^{\prime} E_{\gamma}^{\prime} /\left(m_{p} m_{e} c^{4}\right)\right]-106 / 9\right\}$. The logarithmic increase of the crosssection with incident proton energy implies that this is a very efficient cooling mechanism for high energy protons. The $e^{+} e^{-}$pairs are produced at rest in the center of mass (c.m.) frame of the collision and acquire an energy $m_{e} c^{2} \gamma_{\text {c.m. }}^{\prime}$ each in the comoving frame. Here $\gamma_{\mathrm{c} . \mathrm{m} .}^{\prime}=\left(E_{p}^{\prime}+E_{\gamma}^{\prime}\right) /\left(m_{p}^{2} c^{4}+2 E_{p}^{\prime} E_{\gamma}^{\prime}\right)^{1 / 2}$ is the Lorentz boost factor of the c.m. in the comoving frame. The energy lost by the proton in each $\mathrm{BH}$ interaction is thus the energy of the created pairs in the comoving frame, and is given by $\Delta E_{p}^{\prime}=2 m_{e} c^{2} \gamma_{\text {c.m. }}^{\prime}$. The energy loss rate of the proton is proportional to the $\mathrm{BH}$ scattering rate as given by $d E_{p}^{\prime} / d t_{\mathrm{BH}}^{\prime}=n_{\gamma}^{\prime} c \sigma_{\mathrm{BH}} \Delta E_{p}^{\prime}$, and the corresponding proton cooling time is

$$
t_{\mathrm{BH}}^{\prime}=\frac{E_{p}^{\prime}}{d E_{p}^{\prime} / d t_{\mathrm{BH}}^{\prime}}=\frac{E_{p}^{\prime}\left(m_{p}^{2} c^{4}+2 E_{p}^{\prime} E_{\gamma}^{\prime}\right)^{1 / 2}}{2 n_{\gamma}^{\prime} \sigma_{\mathrm{BH}} m_{e} c^{3}\left(E_{p}^{\prime}+E_{\gamma}^{\prime}\right)},
$$

in the comoving frame.

\subsection{Hadronic cooling channels}

Photomeson $(p \gamma)$ and proton-proton $(p p)$ interactions which are responsible for producing high energy neutrinos may also serve as a cooling mechanism for the shock accelerated protons. The $p \gamma$ at the $\Delta^{+}$resonance and the average $p p$ crosssections are $\sigma_{p \gamma}=5 \times 10^{-28} \mathrm{~cm}^{2}$ and $\sigma_{p p} \approx 5 \times 10^{-26} \mathrm{~cm}^{2}$ respectively. The corresponding optical depths, given by

$$
\begin{aligned}
& \tau_{p \gamma}^{\prime}=\frac{\sigma_{p \gamma} n_{\gamma}^{\prime} r_{j}}{\Gamma_{b}} \approx 10^{7.8}\left(\frac{\varepsilon_{e,-1}^{3} E_{51.5}^{3}}{\Gamma_{b, 0.5}^{8} t_{j, 1}^{3} t_{v,-1}^{2}}\right)^{1 / 4} \text { and } \\
& \tau_{p p}^{\prime}=\frac{\sigma_{p p} n_{p}^{\prime} r_{j}}{\Gamma_{b}} \approx 10^{5.5}\left(\frac{E_{51.5}}{\Gamma_{b, 0.5}^{3} t_{j, 1} t_{v,-1}}\right),
\end{aligned}
$$

are very high. The threshold proton energy for $\Delta^{+}$production against the target thermal photons of energy $E_{\gamma}^{\prime}$ in Eq. (4) is

$$
E_{p, \Delta^{+}}^{\prime}=\frac{0.3 \mathrm{GeV}^{2}}{E_{\gamma}^{\prime}} \approx 10^{4.8}\left(\frac{\Gamma_{b, 0.5}^{4} t_{j, 1} t_{v,-1}^{2}}{\varepsilon_{e,-1} E_{51.5}}\right)^{1 / 4} \mathrm{GeV}
$$

Adopting the energy loss by a proton $\Delta E_{p}^{\prime} \approx 0.2 E_{p}^{\prime}$ and $0.8 E_{p}^{\prime}$ respectively for each $p \gamma$ and $p p$ interaction, the hadronic cooling time scales are

$$
t_{p \gamma}^{\prime}=\frac{E_{p}^{\prime}}{c \sigma_{p \gamma} n_{\gamma}^{\prime} \Delta E_{p}^{\prime}} \approx 10^{-7.3}\left(\frac{\Gamma_{b, 0.5}^{12} t_{j, 1}^{3} t_{v,-1}^{6}}{\varepsilon_{e,-1}^{3} E_{51.5}^{3}}\right)^{1 / 4} \mathrm{~s}
$$




$$
t_{p p}^{\prime}=\frac{E_{p}^{\prime}}{c \sigma_{p p} n_{p}^{\prime} \Delta E_{p}^{\prime}} \approx 10^{-5.6}\left(\frac{\Gamma_{b, 0.5}^{4} t_{j, 1} t_{v,-1}^{2}}{E_{51.5}}\right) \mathrm{s},
$$

using Eqs. (5) and (11). The photomeson cooling time scale $t_{p \gamma}^{\prime}$ above is roughly valid at $E_{p}^{\prime}=E_{p, \Delta^{+}}^{\prime}$ and one needs to use the thermal photon spectrum to calculate it for different proton energies.

\subsection{Maximum proton energy}

The shock acceleration time in Eq. (6) for protons in the SN jet (solid line) and the different cooling time scales are plotted in Fig. 2 as functions of the comoving proton energy. The dashed lines are hadronic cooling time scales in Eq. (12) ${ }^{c}$ and the dot-dashed lines are electromagnetic cooling time scales in Eqs. (77), (8) and (92). Note that the hadronic cooling time $\left(t_{p p}^{\prime}\right)$, the $\mathrm{BH}$ cooling time $\left(t_{\mathrm{BH}}^{\prime}\right)$ and the synchrotron cooling time $\left(t_{\mathrm{syn}}^{\prime}\right)$ are first longer and then shorter than the maximum proton acceleration time $\left(t_{\mathrm{acc}}^{\prime}\right)$. The hadronic $\left(t_{p \gamma}^{\prime}\right)$ and IC scattering $\left(t_{\mathrm{IC}}^{\prime}\right)$ are not efficient cooling mechanisms for protons. The maximum proton energy can be roughly estimated, by equating the $t_{\mathrm{syn}}^{\prime}$ to $t_{\mathrm{acc}}^{\prime}$, from Eqs. (6) and (7) as

$$
E_{p, \max }^{\prime}=\left(\frac{6 \pi m_{p}^{4} c^{4} q \Gamma_{b}^{2}}{\sigma_{\mathrm{Th}} m_{e}^{2} B^{\prime}}\right)^{1 / 2} \approx 10^{6.3}\left(\frac{\Gamma_{b, 0.5}^{4} t_{j, 1} t_{v,-1}^{2}}{\kappa_{1} \varepsilon_{B,-1} E_{51.5}}\right)^{1 / 4} \mathrm{GeV}
$$

since $t_{\mathrm{BH}}^{\prime} \approx t_{\mathrm{syn}}^{\prime} \approx t_{\mathrm{acc}}^{\prime}$ at this energy.

\section{Neutrino Production and Flux on Earth}

Shock accelerated protons in the SN jet can produce non-thermal neutrinos by photomeson $(p \gamma)$ interactions with thermal synchrotron photons and/or by protonproton $(p p)$ interactions with cold protons present in the shock region. As shown in Fig. 22 the $p \gamma$ process is dominant in the energy range $E_{p}^{\prime} \approx 10^{4}-10^{5.2} \mathrm{GeV}$ and the $p p$ process is dominant at other energies.

In the case of $p \gamma$ interactions at the $\Delta^{+}$resonance, neutrinos are produced from charged pion $\left(\pi^{+}\right)$decay as $p \gamma \rightarrow \Delta^{+} \rightarrow n \pi^{+} \rightarrow \mu^{+} \nu_{\mu} \rightarrow e^{+} \nu_{e} \bar{\nu}_{\mu} \nu_{\mu}$. The $p p$ interactions also produce charged pions $\left(\pi^{ \pm}\right)$and kaons $\left(K^{ \pm}\right)$and their decay modes are the same as above with $100 \%$ and $63 \%$ branching ratios respectively. ${ }^{\mathrm{d}}$ The total pion (kaon) multiplicity in each $p p$ interaction is $\sim 1(\sim 0.1)$ in the energy range considered here .35 The energy of the shock accelerated protons in the SN jet is expected to be distributed as $\propto 1 / E_{p}^{\prime 2}$, following the standard shock acceleration models. Charged mesons, produced by $p p$ and $p \gamma$ interactions, are expected to follow the proton spectrum with $\sim 20 \%$ of the proton energy for each pion or kaon.

${ }^{\mathrm{c}} \mathrm{A}$ thermal photon spectrum is used to calculate $p \gamma$ cooling with a delta-function approximation. ${ }^{\mathrm{d}} \pi^{-}$and $K^{-}$decay modes are charge conjugate of $\pi^{+}$and $K^{+}$decay modes. 


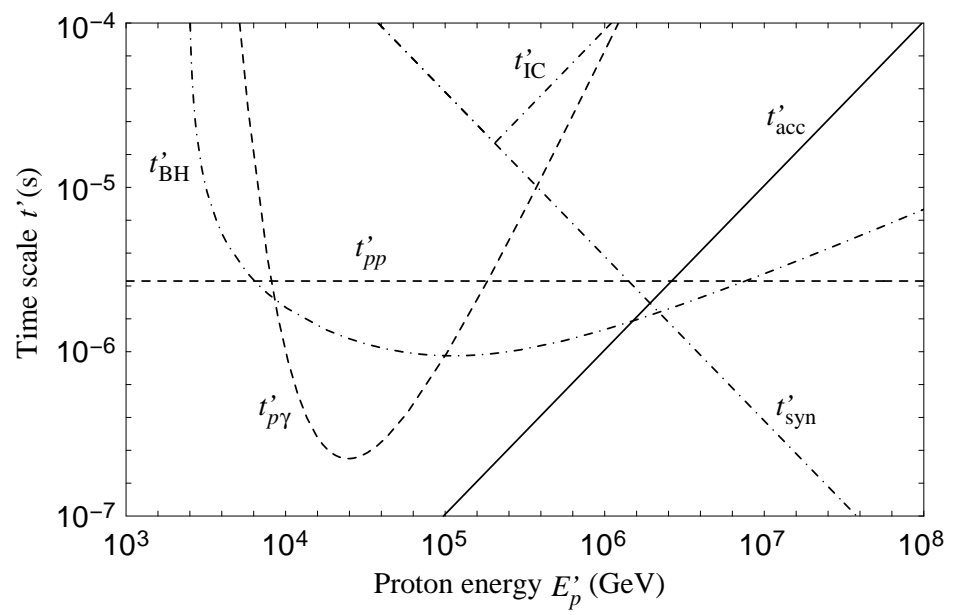

Fig. 2. Proton cooling time scales for different hadronic (dashed lines) and electromagnetic (dotdashed lines) processes: photomeson $\left(t_{p \gamma}^{\prime}\right)$, proton-proton $\left(t_{p p}^{\prime}\right)$, synchrotron radiation $\left(t_{\mathrm{syn}}^{\prime}\right)$, IC scattering $\left(t_{\mathrm{IC}}^{\prime}\right)$ and $\mathrm{BH}\left(t_{\mathrm{BH}}^{\prime}\right)$; in the comoving frame, as functions of energy. The shock acceleration time $\left(t_{\mathrm{acc}}^{\prime}\right)$ is plotted with a solid line. The parameters used to model the SN jet are $E_{\mathrm{sn}}=10^{51.5} \mathrm{erg}, t_{j}=10 \mathrm{~s}, t_{v}=10^{-1} \mathrm{~s}, \Gamma_{b}=10^{0.5}$ and $\varepsilon_{e}=\varepsilon_{B}=0.1$.

\subsection{Meson suppression factors}

High-energy pions, kaons and muons produced by $p \gamma$ and $p p$ interactions do not all decay to neutrinos as electromagnetic (synchrotron radiation and IC scattering) and hadronic ( $\pi p$ and $K p$ interactions) cooling mechanisms reduce their energy. Muons are severely suppressed by electromagnetic energy losses and do not contribute much to high-energy neutrino production. Suppression factors for pion and kaon decay neutrinos are discussed next.

The synchrotron and IC cooling times may be combined into a single electromagnetic cooling rate as $t_{\mathrm{em}}^{\prime-1}=t_{\mathrm{syn}}^{\prime-1}+t_{\mathrm{IC}}^{\prime-1}$. For IC cooling in the Thomson regime $t_{\mathrm{IC}}^{\prime} \approx t_{\mathrm{syn}}^{\prime}$ and in the $\mathrm{KN}$ regime $t_{\mathrm{IC}}^{\prime} \gg t_{\mathrm{syn}}^{\prime}$. The electromagnetic cooling time scales for mesons may be estimated assuming $t_{\mathrm{em}}^{\prime} \approx t_{\mathrm{syn}}^{\prime}$ for simplicity as

$$
t_{\mathrm{em}}^{\prime} \approx\left\{\begin{array}{l}
0.002\left(E_{\pi}^{\prime} / \mathrm{GeV}\right)^{-1} \\
0.3\left(E_{K}^{\prime} / \mathrm{GeV}\right)^{-1}
\end{array} \times\left(\frac{\Gamma_{b, 0.5}^{4} t_{j, 1} t_{v,-1}^{2}}{\varepsilon_{B,-1} E_{51.5}}\right) \mathrm{s} .\right.
$$

The hadronic energy losses for mesons is similar to the proton energy losses by $p p$ interactions in Eq. (12) with the same $\pi p$ and $K p$ cross-section of $\approx 3 \times 10^{-26} \mathrm{~cm}^{2}$. The corresponding hadronic cooling time scales for mesons are

$$
t_{\text {had }}^{\prime} \approx 10^{-5.4}\left(\frac{\Gamma_{b, 0.5}^{4} t_{j, 1} t_{v,-1}^{2}}{E_{51.5}}\right) \mathrm{s},
$$

with $\Delta E^{\prime}=0.8 E^{\prime}$.

The electromagnetic $\left(t_{\pi ; K, \mathrm{em}}^{\prime}\right)$ and hadronic $\left(t_{\text {had }}^{\prime}\right)$ cooling time scales for mesons along with their decay times boosted by the respective Lorentz factors, $t_{\pi ; K, \mathrm{dec}}^{\prime}$, are 


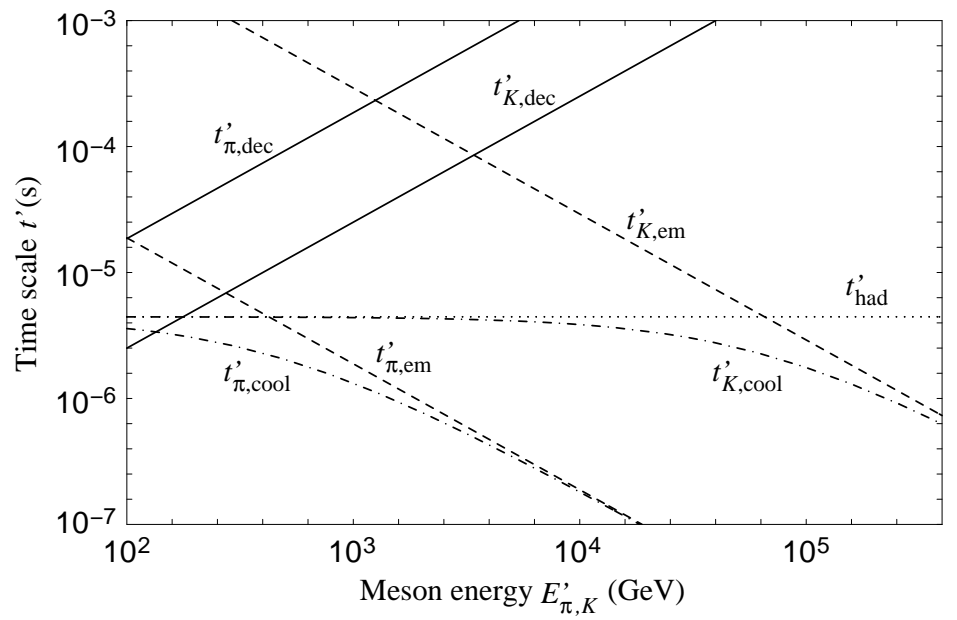

Fig. 3. The Hadronic $\left(t_{\text {had }}^{\prime}\right)$, electromagnetic $\left(t_{\pi ; K, \mathrm{em}}^{\prime}\right)$ and total $\left(t_{\pi ; K, \mathrm{cool}}^{\prime}\right)$ cooling time scales for mesons: $\pi$ and $K$ as functions of comoving energy in a SN jet model by dashed, dotted and dot-dashed lines respectively. The meson decay time scales boosted by the respective Lorentz factors $\left(t_{\pi ; K, \mathrm{dec}}^{\prime}\right)$ are also plotted with solid lines. The parameters used to model the SN jet are $E_{\mathrm{sn}}=10^{51.5} \mathrm{erg}, t_{j}=10 \mathrm{~s}, t_{v}=10^{-1} \mathrm{~s}, \Gamma_{b}=10^{0.5}$ and $\varepsilon_{e}=\varepsilon_{B}=0.1$.

plotted in Fig. 3. The total cooling time scale $t_{\pi ; K, \text { cool }}^{\prime}=1 /\left(t_{\pi ; K, \text { em }}^{\prime-1}+t_{\pi ; K, \text { had }}^{\prime-1}\right)$ is first dominated by the hadronic and then by the electromagnetic cooling channel. The ratio $t_{\pi ; K, \mathrm{cool}}^{\prime} / t_{\pi ; K, \mathrm{dec}}^{\prime}$ determines the suppression of mesons before they decay to neutrinos.

From the condition $t_{\mathrm{em}}^{\prime}=t_{\text {had }}^{\prime}$, one may roughly define a break energy as

$$
E_{\mathrm{br}}^{\prime}= \begin{cases}423 / \varepsilon_{B,-1} \mathrm{GeV} & \text { (pion) } \\ 6.5 \times 10^{4} / \varepsilon_{B,-1} \mathrm{GeV} & \text { (kaon) },\end{cases}
$$

above and below which the mesons cool by electromagnetic and hadronic interactions respectively. The corresponding suppression factor may be defined from the ratios $t_{\mathrm{em}}^{\prime} / t_{\mathrm{dec}}^{\prime}$ and $t_{\mathrm{had}}^{\prime} / t_{\mathrm{dec}}^{\prime}$ as

$$
\zeta=\left\{\begin{array}{l}
10^{-1.25}\left(E_{\pi}^{\prime} / E_{\pi, \mathrm{br}}^{\prime}\right)^{-\beta} \varepsilon_{B,-1} \\
10^{-2.57}\left(E_{K}^{\prime} / E_{K, \mathrm{br}}^{\prime}\right)^{-\beta} \varepsilon_{B,-1}
\end{array} ; \beta=\left\{\begin{array}{l}
1 ; E_{\pi, K}^{\prime}<E_{\pi ; K, \mathrm{br}}^{\prime} \\
2 ; E_{\pi, K}^{\prime}>E_{\pi ; K, \mathrm{br}}^{\prime} .
\end{array}\right.\right.
$$

Another break energy may be defined from the condition $t_{\text {dec }}^{\prime}=t_{\text {had }}^{\prime}$ below which mesons decay to neutrinos without any suppression. However, this energy: 24 and $177 \mathrm{GeV}$ respectively for pions and kaons in the comoving frame is low. The observed energy on Earth in both cases is below the detection threshold energy as will be discussed shortly. 


\subsection{Neutrino fluence from individual sources}

If the shock accelerated protons could travel unimpeded from the SN jet at a luminosity distance $D_{L}$, then their isotropic equivalent fluence on Earth would be ${ }^{\mathrm{e}}$

$$
\mathcal{F}_{p, \mathrm{ob}}=\frac{E_{j} \Gamma_{b}^{2}(1+z)}{2 \pi D_{L}^{2} E_{p, \mathrm{ob}}^{2} \ln \left[E_{p, \mathrm{ob}, \max } / E_{p, \mathrm{ob}, \min }\right]} .
$$

Here $E_{p, \mathrm{ob}}=E_{p} /(1+z)$ and $t_{j, \mathrm{ob}}=t_{j}(1+z)$ are the energy and time related in the observer's and local rest frames for the source location at redshift $z$. Of course, defelction by magnetic fields as well as other interactions along the way prevent such direct arrivale of the protons.

Nearly all protons are expected to convert into $\pi^{+}\left(\pi^{ \pm}\right)$by $p \gamma(p p)$ interactions in the SN jet because $\tau_{p \gamma}^{\prime} \gg 1\left(\tau_{p p}^{\prime} \gg 1\right)$ in Eq. (10) and assuming the charged pion multiplicity is $\sim 1$ from $p p$ interactions as a conservative estimate. With a $100 \%$ branching ratio for pion decay to neutrinos, one may define a multiplicative factor $f_{\pi} \sim 1$ for protons which will produce neutrinos via pions. A similar factor $f_{K} \sim 0.063$ may be defined for kaons as a product of $K^{ \pm}$multiplicity of $\sim 0.1$ from $p p$ interactions and $63 \%$ branching ratio for kaon decay to neutrinos. Protons produce neutral pions (kaons) and charged pions (kaons) with equal probability in both the $p \gamma$ and $p p$ interactions. ${ }^{\mathrm{f}}$ For simplicity one may also assume that a $\nu_{\mu}$ carries $1 / 4$ of the charged pion (kaon) energy: $E_{\nu} \approx E_{\pi, K} / 4$, from roughly equipartition of energy between the final decay products of $\pi^{ \pm}$and $K^{ \pm}$. From these considerations one may estimate the neutrino (of one flavor) fluence on Earth as

$$
\mathcal{F}_{\nu, \mathrm{ob}}=\frac{1}{8} \frac{f_{\pi, K} \eta_{\pi, K} E_{j} \Gamma_{b}^{2}(1+z)}{2 \pi D_{L}^{2} E_{\nu, \mathrm{ob}}^{2} \ln \left[E_{\nu, \mathrm{ob}, \max } / E_{\nu, \mathrm{ob}, \mathrm{min}}\right]}\left(\frac{E_{\nu, \mathrm{ob}}[1+z]}{E_{\nu, \pi ; K, \mathrm{br}}}\right)^{-\beta},
$$

per SN burst using Eqs. (17) and (18). Here $\beta=2$ (1) for $E_{\nu, \mathrm{ob}}(1+z)$ greater (less) than $E_{\nu, \pi ; K, \mathrm{br}}=\Gamma_{b} E_{\pi ; K, \mathrm{br}}^{\prime} / 4$ from Eq. (16). The pre-factors in Eq. (17) are represented by the parameters $\eta_{\pi}=10^{-1.25}$ and $\eta_{K}=10^{-2.57}$ respectively for pions and kaons. For a typical ice Cherenkov detector such as IceCube, the threshold neutrino detection energy is $E_{\nu, \mathrm{th}} \sim 10^{2.5} E_{\nu, 2.5} \mathrm{GeV}$. The neutrino energy range is then $E_{\nu, \mathrm{ob}, \min }-E_{\nu, \mathrm{ob}, \max }=E_{\nu, \mathrm{th}}-0.05 \Gamma_{b} E_{p, \max }^{\prime} /(1+z) \approx 10^{2.5}-10^{5.5} /(1+z)$ $\mathrm{GeV}$ in the observer's frame on Earth.

As mentioned earlier, $\pi^{+}$'s are produced by $p p$ or $p \gamma$ interactions by shock accelerated protons of all energies. In this case $\pi^{+}$decay $\nu_{\mu}$ fluence from a SN at a distance $\sim 20 \mathrm{Mpc}\left(D_{L} \approx 10^{25.8} \mathrm{~cm}, z \sim 0\right)$, e.g. from the Virgo cluster, would be

$$
\begin{aligned}
\mathcal{F}_{\nu, \pi, \mathrm{ob}}^{*} \approx 10^{-5}\left(\frac{E_{\nu, \mathrm{ob}}}{10^{2.5} \mathrm{GeV}}\right)^{-4} \frac{E_{51.5} \Gamma_{b, 0.5}^{2}}{D_{25.8}^{2} \varepsilon_{B,-1}} \mathrm{GeV}^{-1} \mathrm{~cm}^{-2} ; \\
10^{2.5} \lesssim E_{\nu, \mathrm{ob}} / \mathrm{GeV} \lesssim 10^{5.5}
\end{aligned}
$$

eDenoting the quantities in the observer's frame with subscript "ob".

${ }^{\mathrm{f}}$ Kaons are produced by $p p$ interactions only. 
from Eq. (19) with $f_{\pi}=1$. The neutrino break energy from pion decay: $E_{\nu, \pi, \mathrm{br}} \approx$ $E_{\nu, \mathrm{th}} \approx 10^{2.5} \mathrm{GeV}$ in this case. A similar expression may be derived for neutrino fluence from kaon decays. However, $p p$ interactions are overwhelmed by $p \gamma$ interactions in the energy range $E_{p}^{\prime} \approx 10^{4}-10^{5.2} \mathrm{GeV}$ (Fig. 22). Hence kaon and the corresponding decay neutrino production is expected to be suppressed in the energy range $E_{\nu} \approx 10^{3.9}-10^{5.1} \mathrm{GeV}$. The neutrino break energy from Eq. (16) is $E_{\nu, K, \mathrm{br}} \approx 10^{4.7}$ $\mathrm{GeV}$ and the fluence is

$$
\begin{array}{r}
\mathcal{F}_{\nu, K, \mathrm{ob}}^{*} \approx 10^{-11.7}\left(\frac{E_{\nu, \mathrm{ob}}}{10^{4.7} \mathrm{GeV}}\right)^{-(\beta+2)} \frac{E_{51.5} \Gamma_{b, 0.5}^{2}}{D_{25.8}^{2} \varepsilon_{B,-1}^{\beta-1}} \mathrm{GeV}^{-1} \mathrm{~cm}^{-2} ; \\
\beta=\left\{\begin{array}{l}
1 ; 10^{2.5} \lesssim E_{\nu, \mathrm{ob}} / \mathrm{GeV} \lesssim 10^{3.9} \\
2 ; 10^{5.1} \lesssim E_{\nu, \mathrm{ob}} / \mathrm{GeV} \lesssim 10^{5.5},
\end{array}\right.
\end{array}
$$

on Earth from kaon decays in a $\mathrm{SN}$ jet at a distance $\sim 20 \mathrm{Mpc}$.

\subsection{Diffuse neutrino flux}

The diffuse neutrino flux is calculated by summing over fluences from all slow-jet endowed SNe distributed over cosmological distances in Hubble time. The SNe rate follows closely the star formation rate (SFR) which can be modeled, as a function of redshift per unit comoving volume, $\frac{29]}{2}$ as

$$
\dot{\rho}_{*}(z)=\frac{0.32 h_{70} \exp (3.4 z)}{\exp (3.8 z)+45} M_{\odot} \mathrm{yr}^{-1} \mathrm{Mpc}^{-3} .
$$

Here $H_{0}=70 h_{70} \mathrm{~km} \mathrm{~s}^{-1} \mathrm{Mpc}^{-1}$ is the Hubble constant. For a FriedmannRobertson-Walker universe, the comoving volume element is

$$
\frac{d V}{d z}=\frac{4 \pi D_{L}^{2} c}{1+z}\left|\frac{d t}{d z}\right|
$$

and the relation between $z$ and the cosmic time $t$ is

$$
(d t / d z)^{-1}=-H_{0}(1+z) \sqrt{\left(1+\Omega_{m} z\right)(1+z)^{2}-\Omega_{\Lambda}\left(2 z+z^{2}\right)} .
$$

For the standard $\Lambda \mathrm{CDM}$ cosmology, $\Omega_{m}=0.3$ and $\Omega_{\Lambda}=0.7$.

The number of SNe per unit star forming mass $\left(f_{\mathrm{sn}}\right)$ depends on the initial mass function and the threshold for stellar mass to produce $\mathrm{SN}\left(M_{*} \sim 8 \mathrm{M}_{\odot}\right)$. A Salpeter model $\phi(M) \propto M^{-\alpha}$ with different power-law indices can generate different values for $f_{\text {sn }}$, e.g. $\approx 0.0122 \mathrm{M}_{\odot}^{-1}$ for $\alpha=2.35$ and $\approx 8 \times 10^{-3} \mathrm{M}_{\odot}^{-1}$ for $\alpha=1.35 .2930$ We adopt the model in Ref. 29] which corresponds to the local type II SNe rate $\dot{n}_{\mathrm{sn}}(z=0)=f_{\mathrm{sn}} \dot{\rho}_{*}(z=0) \approx 1.2 \times 10^{-4} h_{70}^{3} \mathrm{yr}^{-1} \mathrm{Mpc}^{-3}$ agreeing with data. 31

The distribution of SNe per unit cosmic time $t$ and solid angle $\Omega$ covered on the sky can be written as

$$
\frac{d^{2} N_{\mathrm{sn}}}{d t d \Omega}=\frac{\dot{n}_{\mathrm{sn}}(z)}{4 \pi} \frac{d V}{d z}=\frac{\dot{n}_{\mathrm{sn}}(z) D_{L}^{2} c}{(1+z)^{2}}\left|\frac{d t}{d z}\right| .
$$




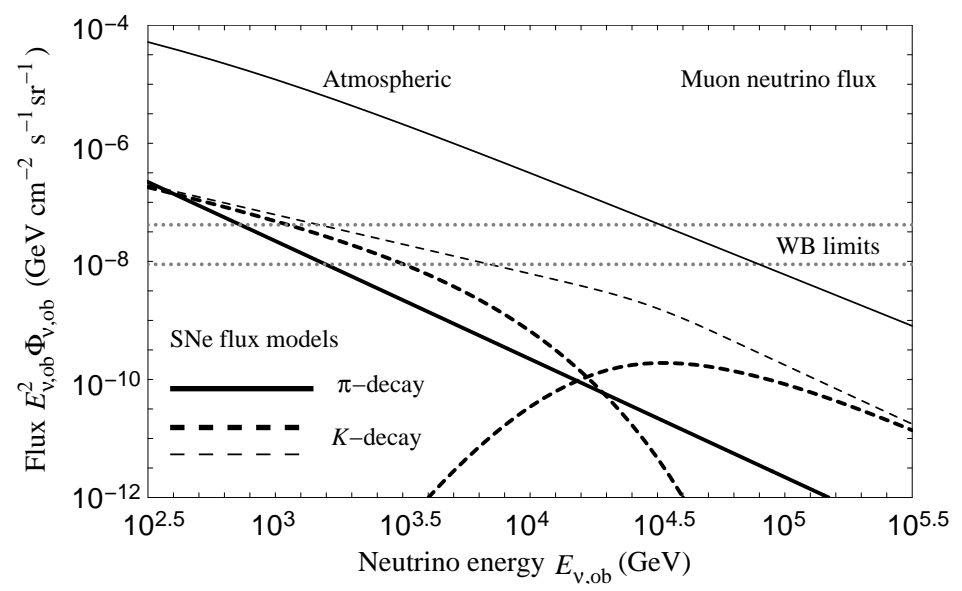

Fig. 4. Diffuse muon neutrino $\left(\nu_{\mu}\right)$ flux models from core collapse SNe assuming all of them $\left[\xi_{\mathrm{sn}}=1\right.$ in Eq. [26] $]$ are endowed with slow jets as discussed here. The pion decay neutrinos are produced via $p p$ and $p \gamma$ interactions over the whole range of shock accelerated proton energy. The kaon decay neutrinos are produced via $p p$ interactions only and may be suppressed (thick dashed line) where $p \gamma$ interactions are dominant over $p p$ interactions. The thin dashed line corresponds to the kaon decay neutrino flux if $p p$ is dominant over the whole proton energy range. The parameters used to model the SN jet are the same as in Figs. 2 and 3 Diffuse fluxes are unlikely to be detected because of the huge atmospheric background.

We assume a fraction $\xi_{\mathrm{sn}} \lesssim 1$ of all SNe involve jets and a fraction $1 / 2 \Gamma_{b}^{2}$ of all such jets are pointing towards us. The observed diffuse SNe neutrino flux, using Eqs. (19) and (25), is then

$$
\begin{aligned}
\Phi_{\nu, \mathrm{ob}}^{\mathrm{diff}}= & \frac{\xi_{\mathrm{sn}}}{2 \Gamma_{b}^{2}} \int_{0}^{\infty} d z \frac{d^{2} N_{\mathrm{sn}}}{d t d \Omega} \mathcal{F}_{\nu, \mathrm{ob}}\left(E_{\nu, \mathrm{ob}}\right) \\
= & \frac{\xi_{\mathrm{sn}} f_{\pi, K} \eta_{\pi, K}}{32 \pi} \frac{c E_{j}}{E_{\nu, \mathrm{ob}}^{2}} \int_{0}^{\infty} d z \frac{\dot{n}_{\mathrm{sn}}(z) /(1+z)}{\ln \left[E_{\nu, \mathrm{ob}, \max } / E_{\nu, \mathrm{th}}\right]}\left|\frac{d t}{d z}\right|\left(\frac{E_{\nu, \mathrm{ob}}[1+z]}{E_{\nu, \pi ; K, \mathrm{br}}}\right)^{-\beta} \\
& \times \Theta\left(E_{\nu, \mathrm{th}} \leq E_{\nu, \mathrm{ob}} \leq E_{\nu, \mathrm{ob}, \max }\right)
\end{aligned}
$$

with $\beta$ specified as before.

The diffuse $\nu_{\mu}$ flux from all cosmological slow-jet SNe is plotted in Fig. 目 by numerically integrating Eq. (26), assuming the maximal fraction $\xi_{\mathrm{sn}}=1$. The pion (kaon) decay flux is plotted with thick solid (dashed) line(s). The exponential suppression in the kaon decay $\nu_{\mu}$ flux curves is due to the lack of kaon production by $p p$ interactions, as $p \gamma$ interactions, which do not produce kaons, are dominant in the particular proton energy range corresponding to this neutrino energy range, $E_{\nu, \mathrm{ob}}(1+z) \approx 10^{3.9}-10^{5.1} \mathrm{GeV}$. The light dashed curve corresponds to the kaon decay $\nu_{\mu}$ flux if $p p$ interaction dominates over the whole proton energy range for comparison. The kaon decay neutrino fluxes are first smaller and then larger than the pion decay neutrino flux. This is because of the kaon's heavier mass and shorter decay time compared to pions (see Fig. 3). 
The atmospheric $\nu_{\mu}, \bar{\nu}_{\mu}$ flux from pion and kaon decays (conventional flux) compatible with AMANDA data $\sqrt{32}$ is plotted with the following parametrization 33

$$
\Phi_{\nu, \mathrm{ob}}^{\mathrm{atm}}=\left\{\begin{array}{l}
0.012 E_{\nu, \mathrm{ob}}^{-2.74} /\left(1+0.002 E_{\nu, \mathrm{ob}}\right) ; E_{\nu, \mathrm{ob}}<10^{5.8} \mathrm{GeV} \\
3.8 E_{\nu, \mathrm{ob}}^{-3.17} /\left(1+0.002 E_{\nu, \mathrm{ob}}\right) ; E_{\nu, \mathrm{ob}}>10^{5.8} \mathrm{GeV}
\end{array}\right.
$$

Also shown are the cosmic ray bounds (WB limits) on the diffuse neutrino flux 17 It is unlikely that neutrino detectors can measure the SNe diffuse fluxes plotted in Figure 4 as they are below the atmospheric background. However, individual SNe in nearby galaxies may be detectable, as discussed in the next section.

\section{Neutrino Detection Prospects}

There are approximately 4000 galaxies known within $20 \mathrm{Mpc}$ distance. At the standard rate of $1 \mathrm{SNu}=10^{-2} / \mathrm{yr} / 10^{10}$ blue solar luminosity for average galaxies, the estimated SN rate is $\gtrsim 1 / \mathrm{yr}$. The SN rate in the starburst galaxies, such as M82 and NGC253 (at distances 3.2 and 2.5 Mpc in the Northern and Southern sky, respectively) is $\sim 0.1 / \mathrm{yr}$, much larger than in the Milkyway or in the Magellanic clouds. ${ }^{[34}$ Very strong neutrino signals in future kilometer scale neutrino detectors are expected from these nearby $\mathrm{SNe}$, over a negligible atmospheric background, using temporal and positional coincidences with optical detections.

The directional sensitivity of the Cherenkov detectors is best for muon neutrinos, which create muons by charge current neutrino-nucleon $(\nu N \rightarrow \mu X)$ interactions. Muons carry $\sim 80 \%$ of the incident neutrino energy. It emits Cherenkov light as it travels faster than the speed of light in the detection media. Photo multiplier tubes (PMTs) buried in the medium (ice, e.g. in the case of IceCube) can detect muons by gathering their Cherenkov light. The effective detection area of a Cherenkov detector depends on the arrival direction of the neutrino and the energy of the muon. In principle it can be larger than the geometrical area $\left(A_{\mathrm{det}} \sim \mathrm{km}^{2}\right.$ in the case of IceCube e.g.) as muons can be produced outside the instrumented volume

and travel inside. To achieve a good pointing resolution a muon should hit at least 4 PMTs, for reconstructing its track unamiguously, strung on different vertical strings. The PMT spacing is $17 \mathrm{~m}$ vertically and $125 \mathrm{~m}$ horizontally. With a muon energy loss of $\sim 0.2 \mathrm{GeV} / \mathrm{m}$ and a PMT efficiency of $\sim 30 \%$, the threshold energy for muon detection is $(3 \times 125 \mathrm{~m}) \times(0.2 \mathrm{GeV} / \mathrm{m}) / 0.3 \approx 250 \mathrm{GeV}$ as a conservative estimate. This corresponds to a neutrino threshold energy $E_{\nu, \mathrm{th}} \approx 10^{2.5} \mathrm{GeV}$ as used before. The IceCube detector is expected to have an angular sensitivity of $1^{\circ}$ for muon tracks of energy $\lesssim 1 \mathrm{TeV}$ coming from a zenith angle $<140^{\circ}$ and it gets better at higher energy! 24

The number of muon events from a nearby jetted SN may be calculated as

$$
N_{\mu}=A_{\operatorname{det}} \int_{E_{\nu, \text { th }}}^{E_{\nu, \max }} \mathcal{P}\left(E_{\nu}, \theta\right) \mathcal{F}_{\nu, \pi ; K}^{*}\left(E_{\nu}\right) d E_{\nu}
$$

where the neutrino fluences are given in Eqs. (20) and (21). The full detection probability $\mathcal{P}\left(E_{\nu}, \theta\right)$ depends on the source's angular position $(\theta)$ and hence on the 


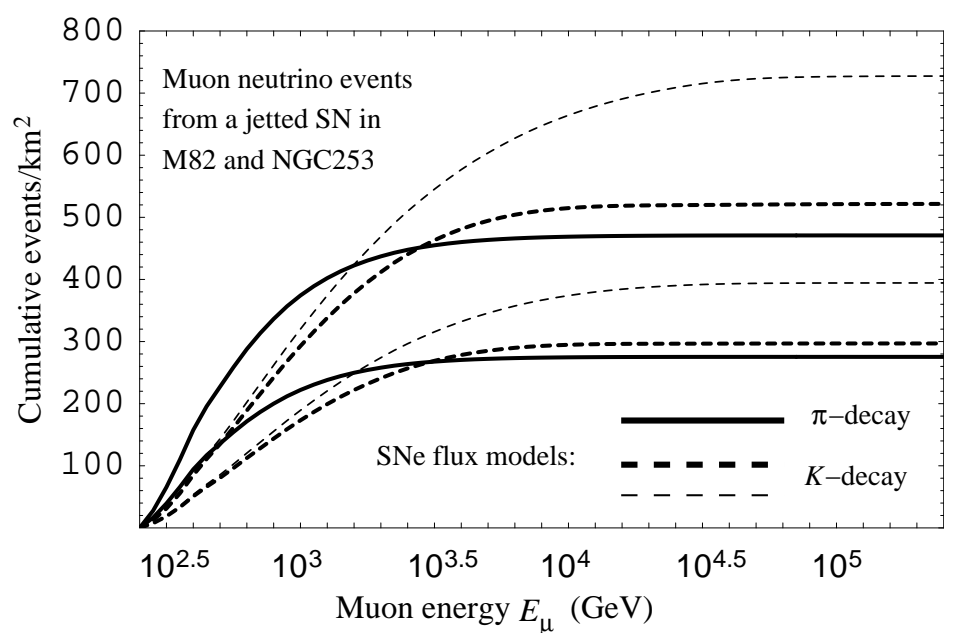

Fig. 5. Cumulative muon events from $\nu_{\mu}$ flux models from a jetted core collapse SN in M82 and NGC253 (lower and upper sets of lines) as functions of observed muon energy in IceCube. The pion and kaon decay neutrino flux models are described in Eqs. [20, 21] and in the caption of Fig. (4) (also in the main text). The neutrino events from pion decay flux models are larger (smaller) than the kaon decay flux models at lower (higher) energy. This is because more pions are produced than kaons but pion decay to neutrino is suppressed due to pion energy loss at higher energy compared to kaons with heavier mass and shorter decay time (Fig. 3).

Earth's shadowing effect, as well as the energy dependent $\nu N$ cross section. .55 The cumulative number of muon events from M82 and NGC253 are plotted in Fig. 5 per individual SN with its jet pointing towards Earth . The $\nu_{\mu}$ flux models are from pion (solid lines) and kaon (dashed lines) decays in the SN jet as in Fig. 4 The two kaon decay models are due to the protons in the SN jet producing kaons in their whole (thick dashed lines) and partial (thin dashed lines) energy range (see Fig. 21). The lower and upper sets of lines at $E_{\mu}=10^{3} \mathrm{GeV}$ correspond to the SN in M82 and NGC253 respectively. The timing uncertainty in the optical detection of SN is $\sim 1$ day. The corresponding atmospheric background events within $1^{\circ}$ angular resolution is 0.07 for both M82 and NGC253 in the same energy range as in Fig. 5

Neutrino fluxes of all three flavors $\left(\nu_{\mu}, \nu_{e}, \nu_{\tau}\right)$ on Earth should be equal because of oscillations over astrophysical distances. However, only $\nu_{\mu}$ 's are emitted from the sources under consideration and the total number of neutrino events (including $\nu_{e}$ and $\nu_{\tau}$ ) will remain the same as the $\nu_{\mu}$ events calculated here. The lack of good directional sensitivity for the $\nu_{e}$ and $\nu_{\tau}$ events may prevent obtaining their positional coincidence with the SN. Timing coincidences of $\nu_{e}$ and $\nu_{\tau}$ events with $\nu_{\mu}$ events, however, may still be useful to verify the neutrino oscillations at these energies and test their common origin.

At the quoted rate for M82 and NGC253, a SN from one of these galaxies would be expected within five years. However, only $1 / 5$ of them would be visible in neutrinos due to the $1 / 2 \Gamma_{b}^{2}$ beaming effect of the jet. IceCube may detect one 
such SN in 25 years of its operation. The real situation is not as pessimistic as for the brightest neutrino events expected from M82 and NGC253. Other nearby spiral galaxies (M31, M74, M51, M101, etc, and the Virgo cluster) will also have SNe. For a hypothetical SN at $20 \mathrm{Mpc}$ with its jet pointing towards Earth, the number of neutrino events is $\approx 15 / \mathrm{km}^{2}$ from pion and kaon decay fluxes combined. With the suggested rate of $\gtrsim 1 \mathrm{SN} / \mathrm{yr}$ within $20 \mathrm{Mpc}$, IceCube may detect $\gtrsim 3$ muon events/yr after beaming correction from the jetted SNe.

\section{Conclusions}

While a core collapse $\mathrm{SN}$ in a typical galaxy is a rare event, their rate of occurence within $20 \mathrm{Mpc}$ could be more than $1 / \mathrm{yr}$, and the physics and astrophysics one can learn from such an explosion is enormous. A buried slow jet from the collapsing core of the supernova progenitor star is an attractive possibility for solving the long standing problem of how to understand the ejection of the envelope in SN explosions, by re-energizing the shock wave through energy deposition by the jet. Alternatively it could be that only a fraction of core collapses leads to such jets. These hypothetical mildly relativistic jets in SNe may be related to the ultra relativistic jets thought to be responsible for long duration GRBs, which are thought to originate from the core collapse of massive progenitor stars, some of which have been positively associated with observed envelope ejection supernova events. While all typical core collapses should produce $10 \mathrm{MeV}$ thermal neutrinos, the presence of a jet would allow proton acceleration by shocks, and produce $1 \mathrm{TeV}$ non-thermal neutrinos. Detection of these high energy neutrinos by upcoming Cherenkov detectors would be a smoking gun signal of a SN jet, and would allow one to study the conditions inside a collapsing star which may not be possible otherwise.

\section{Acknowledgments}

Work supported by an NSF grant AST0307376 and NASA grant NAG5-13286.

\section{References}

1. For a recent review see: M. Della Valle, arXiv:astro-ph/0504517

2. A. MacFadyen and S. E. Woosley, Astrophys. J. 524, 262 (1999) arXiv:astro-ph/9810274.

3. E. Berger, S. R. Kulkarni, D. A. Frail and A. M. Soderberg, Astrophys. J. 599, 408 (2003) arXiv:astro-ph/0307228.

4. J. S. Bloom, S. R. Kulkarni, F. A. Harrison, T. Prince, E. S. Phinney and D. A. Frail, Astrophys. J. 506, L105 (1998) arXiv:astro-ph/9807050.

5. B. M. S. Hansen, Astrophys. J. 512, L117 (1999).

6. A. I. Macfadyen, S. E. Woosley and A. Heger, Astrophys. J. 550, 410 (2001).

7. E. Waxman and A. Loeb, Phys. Rev. Lett. 87, 071101 (2001) arXiv:astro-ph/0102317.

8. E. Ramirez-Ruiz, A. Celotti and M. J. Rees, Mon. Not. Roy. Astron. Soc. 337, 1349 (2002) arXiv:astro-ph/0205108. 
9. J. Granot and A. Loeb, Astrophys. J. 593, L81 (2003) arXiv:astro-ph/0305379.

10. K. Nomoto, P. A. Mazzali, T. Nakamura, K. Iwamoto, I. J. Danziger, F. Patat, in Supernovae and gamma-ray bursts: the greatest explosions since the Big Bang, May, 1999, eds. M. Livio, N. Panagia and K. Sahu. STScI symposium series, Vol. 13, pg 144 (2001).

11. J. Granot and E. Ramirez-Ruiz, Astrophys. J. 609, L9 (2004) arXiv:astro-ph/0403421.

12. L. Wang, D. A. Howell, P. Höflich, and J. C. Wheeler, Astrophys. J. 550, 1030 (2001).

13. D. C. Leonard, A. V. Filippenko, A. J. Barth and T. Matheson Astrophys. J. 536, 239 (2000).

14. For a recent review see: A. Mezzacappa, arXiv:astro-ph/0010580

15. A. M. Khokhlov, P. A. Höflich, E. S. Oran, J. C. Wheeler, L. Wang, and A. Yu. Chtchelkanova, Astrophys. J. 524, L107 (1999).

16. B. Zhang and P. Mészáros, Int. J. Mod. Phys. A 19, 2385 (2004) arXiv:astro-ph/0311321.

17. E. Waxman and J. N. Bahcall, Phys. Rev. Lett. 78, 2292 (1997) arXiv:astro-ph/9701231.

18. P. Mészáros and E. Waxman, Phys. Rev. Lett. 87, 171102 (2001) arXiv:astro-ph/0103275.

19. S. Razzaque, P. Mészáros and E. Waxman, Phys. Rev. D 68, 083001 (2003) arXiv:astro-ph/0303505.

20. S. Razzaque, P. Mészáros and E. Waxman, Phys. Rev. Lett. 93, 181101 (2004) [Erratum-ibid. 94, 109903 (2005)] arXiv:astro-ph/0407064.

21. S. Ando and J. F. Beacom, arXiv:astro-ph/0502521

22. K. Hirata et al. [KAMIOKANDE-II Collaboration], Phys. Rev. Lett. 58, 1490 (1987).

23. F. Cei, Int. J. Mod. Phys. A 17, 1765 (2002) arXiv:hep-ex/0202043.

24. J. Ahrens et al. [IceCube Collaboration], Astropart. Phys. 20, 507 (2004) arXiv:astro-ph/0305196.

25. I. Sokalski [ANTARES Collaboration], arXiv:hep-ex/0501003

26. C. L. Fryer and R. Dupuis, in 30 Years of Discovery: Gamma-Ray Burst Symposium. Edited by E. E. Fenimore and M. Galassi. AIP Conference Proceedings, Vol. 727, 371 (2004).

27. W. Zhang, S. E. Woosley and A. I. MacFadyen, Astrophys. J. 586, 356 (2003) arXiv:astro-ph/0207436.

28. S. Razzaque, P. Mészáros and E. Waxman, Phys. Rev. Lett. 90, 241103 (2003) arXiv:astro-ph/0212536.

29. C. Porciani and P. Madau, Astrophys. J. 548, 522 (2001) arXiv:astro-ph/0008294.

30. L. Hernquist and V. Springel, Mon. Not. Roy. Astron. Soc. 341, 1253 (2003) arXiv:astro-ph/0209183.

31. P. Madau, M. Della Valle and N. Panagia, Mon. Not. R. Astron. Soc. 297, 17L (1998) arXiv:astro-ph/9803284.

32. J. Ahrens et al. [AMANDA Collaboration], Phys. Rev. D 66, 012005 (2002) arXiv:astro-ph/0205109.

33. M. Thunman, P. Gondolo and G. Ingelman, Astropart. Phys. 5, 309 (1996) arXiv:hep-ph/9505417.

34. A. Alonso-Herrero et al., Aston. J. 125, 1210A (2003).

35. see e.g. S. Razzaque, P. Mészáros and E. Waxman, Phys. Rev. D 69, 023001 (2004) arXiv:astro-ph/0308239. 